\title{
MERCADOS LABORALES INTERNOS \\ EN LA COMPAÑÍA FERROVIARIA MZA, 1882-1889*
}

\author{
FABIOLA PORTILLO PÉREZ DE VIÑASPRE \\ Universidad de La Rioja ${ }^{\text {a }}$ \\ CECILIO MAR MOLINERO \\ University of Kent ${ }^{\text {b }}$
}

\section{RESUMEN}

En este estudio se analiza la posible existencia de alguna forma incipiente de Mercados Laborales Internos (MLIs) en la compañía ferroviaria MZA, en los comienzos de la segunda revolución industrial. Para ello se aplican los métodos no lineales de Escalado Óptimo. Tal como predice la teoría, los resultados muestran dos rasgos característicos del funcionamiento de dichos mercados: la existencia de "puertos de entrada" de los trabajadores en la compañía para niveles bajos de cualificación profesional y el establecimiento de relaciones laborales en el largo plazo. Por tanto, análogamente a los resultados que presentan recientes estudios sobre empresas ferroviarias del Reino Unido, puede concluirse que en España la compañía MZA ya operaba con una estructura de MLIs antes del siglo XX.

* Received 10/02/2007. Accepted 01/14/2008. Deseamos expresar nuestro agradecimiento al Dr. Tomás Martínez Vara, de la Universidad Complutense de Madrid, que desinteresadamente ha colaborado suministrando información estadística para la elaboración del estudio presentado y, asimismo, a los evaluadores anónimos de la Revista de Historia Económica por sus sugerencias y aportaciones que han mejorado sustancialmente el trabajo. El desarrollo de esta investigación ha sido posible gracias a becas y convenios de movilidad entre la Universidad de La Rioja y la Kent Business School.

a Departamento de Economía y Empresa, C/ Cigüeña 60, 26004 Logrono, fabiola.portillo@unirioja.es.

b Kent Business School, Canterbury, Kent CT2 7PE, United Kingdom, C.Mar-molinero@kent. ac.uk. 
Palabras clave: historia económica del transporte, mercados laborales internos, escalado óptimo, componentes principales categóricos, propertyfitting

\begin{abstract}
The possible existence of Internal Labour Markets (ILMs) in the Spanish railway company MZA at the beginning of the Second Industrial Revolution is explored by means of non-linear Optimal Scaling techniques. The results show the presence of two characteristics of ILMs predicted by the theory: the existence of "ports of entry» for the employees of the company at low levels of qualification, and the presence of long-term labour relations. Thus, in common with recent studies on UK railway companies, we conclude that the company MZA operated some kind of ILM before the start of the $20^{\text {th }}$ Century.
\end{abstract}

Keywords: transport economic history, internal labour markets, optimal scaling, categorical principal components, property-fitting

JEL Classification: C10, J21, N73

\title{
1. INTRODUCCIÓN
}

En las últimas décadas, numerosos trabajos han tratado de evaluar la importancia de las contribuciones del ferrocarril al desarrollo económico español -entre los que cabe destacar los trabajos seminales de Casares (1973), Tortella (1973), Nadal (1975), Anes (1978), Artola (1978), Tedde (1978) y Gómez Mendoza (1982)—, adoptando para ello diversos enfoques ${ }^{1}$. No obstante, como ponen de relieve Muñoz y Vidal (2001, p. 106), los temas inexplorados en este ámbito son muy amplios, particularmente en lo relativo a la etapa en que el ferrocarril fue gestionado por compañías privadas, ya que estas investigaciones están condicionadas en gran medida por la documentación histórica disponible, así como por el interés que generan en la comunidad científica. A este respecto, Ballesteros y Martínez Vara (2001, p. 638) inciden en la escasez existente de estudios sobre las relaciones laborales y la gestión empresarial en las compañías ferroviarias, con la excepción de las aportaciones realizadas por Soto (1989), Juez (1992), Pérez Sánchez (1992), Ballesteros (1999), Ortúñez (1999) y Vidal (1997, 1999), y más recientemente, Martínez Vara $(2005,2006)$ y Portillo et al. (2006).

${ }^{1}$ Una revisión de esta amplia literatura puede verse en Comín et al. (1998), Muñoz y Vidal (2001) y Herranz (2003). 
En el contexto planteado, y centrándonos en el marco teórico que proporcionan los Mercados Laborales Internos (MLIs), este artículo se dirige a analizar el empleo en la "Compañía de los Caminos de Hierro de Madrid a Zaragoza y Alicante» (MZA) durante el periodo 1882-1889, en plena expansión de esta compañía ferroviaria; en dicho periodo MZA incrementó los kilómetros de ferrocarril en aproximadamente el 37 por cien, siguiendo la tendencia iniciada en 1865 (Anes, 1978). Hasta bien avanzada la segunda década del siglo xx, MZA ocupó la primera y segunda posiciones en la relación de las mayores empresas de nuestro país, y la décima en el ámbito europeo (Carreras y Tafunell, 1996). Así pues, este análisis se dirige a profundizar en el conocimiento de las formas más avanzadas de gestión empresarial aplicadas en España, en los comienzos de la segunda revolución industrial.

En cuanto a los MLIs, Doeringer y Piore $(1971$, p. 37) señalan que, si bien los orígenes de este sistema de gestión empresarial parecen situarse en el siglo XIX, existe una clara necesidad de someter esta hipótesis a contrastación empírica mediante el desarrollo de investigaciones históricas relevantes. Esto es debido a que la mayor parte de la literatura económica en este ámbito se centra en el estudio de los MLIs ya en el siglo Xx. Ahora bien, otros estudios más recientes, como son los desarrollados por Drummond (1989, 1995), Mackinnon (1998), Savage (1998) y Howlett, (2000, 2001, 2004), han realizado interesantes aportaciones en este sentido, presentando indicios de la posible implantación de MLIs por compañías ferroviarias británicas y australianas a finales del siglo XIX. En esta misma línea, cabe plantearse si existía ya alguna forma incipiente de MLIs en la compañía MZA, en ese mismo periodo.

La documentación histórica disponible para realizar este análisis consiste en una base de datos que contiene una muestra de expedientes de personal conservados de trabajadores de la compañía MZA, procedente del Archivo de la Fundación de los Ferrocarriles Españoles. Para cada uno de los empleados, esta base de datos proporciona información sobre la edad en que fueron contratados, el número de años que permanecieron en la empresa y el salario inicial, todas ellas variables de tipo cuantitativo. Además, la base de datos incluye otras características de los empleados, como son estado civil, tipo de trabajo para el que fueron contratados, motivos por los que cesó su relación con la compañía y lugar de nacimiento, todas ellas variables cualitativas. Así pues, para explotar la información que contienen estos datos de naturaleza multivariante, es necesario trabajar conjuntamente con variables cualitativas y cuantitativas, y por tanto medidas en distintos tipos de escalas, lo que condiciona los métodos estadísticos aplicables (ver Stevens, 1951).

El procedimiento seguido consiste en la transformación de los valores cualitativos en cuantitativos mediante los métodos no lineales de Escalado Óptimo (EO). Una vez cuantificadas las variables cualitativas, es posible aplicar a los datos las técnicas estándar del análisis multivariante. En este 
trabajo se ha utilizado el análisis de Componentes Principales Categóricos (CPC) y el análisis de Ajuste de Propiedades (Property-Fitting o Pro-Fit), ya que permiten la interpretación gráfica de los resultados (ver Kruskal y Wish, 1978, y Mar Molinero y Mingers, 2007).

Los resultados obtenidos muestran que los trabajadores de la compañía de nacionalidad extranjera tendían a ser empleados en trabajos más cualificados y, consecuentemente, remunerados con mayores salarios que los trabajadores españoles. También es posible observar que la mayoría de los trabajadores que interrumpieron su relación con la compañía por motivos extraordinarios - dimisión o despido disciplinario- lo hicieron poco tiempo después de entrar a trabajar en la empresa, no observándose relación entre este hecho y el salario inicial, ni tampoco con la categoría profesional en el momento de ser contratados. Estos resultados son consecuentes con la existencia de alguna forma incipiente de MLIs en la compañía ferroviaria MZA, cuestión que será analizada en profundidad en epígrafes posteriores.

El resto del trabajo se ha estructurado del siguiente modo. En primer lugar, se realiza una breve revisión de la literatura económica sobre los principales modelos teóricos que analizan los MLIs. A continuación se presentan los datos empleados en este estudio, para pasar seguidamente a mostrar los resultados obtenidos tras la aplicación de modelo propuesto. Finalmente, se resumen las principales conclusiones del trabajo. Los fundamentos metodológicos del modelo aplicado se describen en un apéndice técnico.

\section{NUEVAS FORMAS DE ORGANIZACIÓN DEL TRABAJO: MERCADOS LABORALES INTERNOS}

Durante la segunda mitad del siglo XIX, el desarrollo de las grandes compañías ferroviarias en Estados Unidos propició la formación de la empresa corporativa y dio origen a la gestión moderna (Chandler, 1977). En Europa, aunque con una menor proyección en la economía y con características específicas para los diferentes países (ver para España Gómez Mendoza, 1982; Comín, 1983, y Vidal, 1997), la contribución de estas compañías a la innovación en las formas de gestión empresarial fue fundamental, al generar y potenciar una cultura de nuevas estructuras organizativas (ver, por ejemplo, Caron, 2003). En este ámbito, España no fue una excepción (Vidal, 1996, 1999). Con el fin de alcanzar una dimensión óptima que les permitiera reducir los costes medios y beneficiarse de las economías de escala, estas empresas vivieron una etapa de expansión acelerada entre 1865 y 1890 (Tedde, 1996, y Tortella 1999), en la que se enfrentaron a la selección y dirección de un número elevado y diversificado de trabajadores, con profesiones en ocasiones nuevas, para cuyo desarrollo se requería una cualifica- 
ción que las propias compañías debían proporcionar ${ }^{2}$ (Chandler, 1965, y Drummond, 1989). Esto supuso una rápida ampliación de sus bases organizativas, jerarquizándose notablemente la toma de decisiones por la creciente complejidad de los organigramas internos de explotación.

En este contexto, la organización del trabajo constituyó un área de interés preferente para los órganos directivos de estas grandes compañías ferroviarias (Mackinnon, 1994) y, como apuntan Fitzgerald (1988) y Drummond (1989), dicha organización se basó por primera vez en empresas corporativas en el desarrollo de extensivos MLIs, que se fundamentó en todo un sistema de programas de bienestar industrial. Aunque la mayor parte de la literatura económica que analiza esta cuestión se centra en el desarrollo de MLIs en las primeras décadas del siglo $\mathrm{xx}$, cuestión sobre la que existe actualmente un amplio consenso, otros estudios más recientes (Drummond, 1989, pp. 10-11; Drummond, 1995, cap. 2-5; Mackinnon, 1998, p. 84; Savage, 1998, pp. 70-72, y Howlett, 2000, pp. 21-22; Howlett, 2001, p. 22, y Howlett, 2004 , p. 420) sugieren su posible origen e implantación por compañías ferroviarias británicas y australianas ya a finales del siglo XIX. En esta misma línea, cabe plantearse si existía ya alguna forma incipiente de MLIs en la compañía MZA, en los comienzos de la segunda revolución industrial.

Un sistema de MLIs constituye todo un conjunto de normas y procedimientos establecidos en el seno de una empresa que regulan las relaciones existentes entre ésta y los recursos humanos, en particular en lo relativo a los puestos de entrada de los trabajadores en la empresa, los procesos de promoción interna y el sistema de remuneración de los empleados (Lazear y Oyer, 2003). En un MLI la entrada de nuevos trabajadores a la empresa se produce principalmente en niveles bajos de cualificación profesional, denominados "puertos de entrada» (Doeringer y Piore, 1971). Coherentemente con los planteamientos teóricos del modelo de costes de transacción (Wachter y Wright, 1990, y Siebert y Addison, 1991), el acceso a los puestos de trabajo de mayor nivel estaría limitado a los empleados con antigüedad en la empresa, con el fin de retener a los trabajadores más cualificados mediante la promoción interna. En cuanto al sistema de remuneración de los empleados, que puede ser analizado desde el enfoque del modelo de salarios de eficiencia (Idson y Feaster, 1990), éste se basa en salarios altos con respecto a los vigentes en el mercado exterior que en parte se materia-

2 En este sentido, Drummond (1989, p. 13) pone de relieve que en 1881 los trabajadores de los talleres de Crewe y Derby, en el Reino Unido, habían sido formados en sus especialidades predominantemente por las propias companías ferroviarias. Este es un aspecto diferencial en la formación de los trabajadores con respecto a épocas anteriores, deducido a partir de los censos de población - Census Enumerator-. En cuanto al diseño de políticas de captación y disciplina de una fuerza de trabajo tan ingente, Drummond $(1989$, p. 11) sugiere que, para el caso británico, las companías ferroviarias contaron con importantes precedentes, como son, la Companía Británica de las Indias Orientales y la Armada Británica, organizaciones que procuraron a dichas companías muchos de sus primeros managers o gestores. 
lizan en beneficios derivados de programas de bienestar industrial, más o menos sistematizados. Así pues, según estos modelos teóricos, las salidas y entradas en la empresa se producirían en los niveles más bajos, ya que quienes acumulan cierta antigüedad en la empresa se beneficiarían de las ventajas salariales y sociales de los MLIs, que perderían si abandonaran su puesto de trabajo. Por ejemplo, desde 1874 MZA había establecido una Caja de Previsión que requería un mínimo de cinco años de cotización antes de proporcionar beneficios a los empleados de plantilla, maquinistas y fogoneros $^{3}$ (Comín et al., 1998).

El desarrollo de un MLI implica la internalización dentro de la empresa de procesos laborales que habitualmente se llevan a cabo externamente. Dicha internalización está asociada al establecimiento de organizaciones modernas que operan a gran escala en mercados bien definidos y relativamente estables, y que por tanto pueden permitirse contratar en el largo plazo, entre las que son pioneras las grandes compañías ferroviarias (Howlett, 2000). En este sentido, diversos estudios, tanto teóricos como empíricos, muestran que únicamente las empresas que operan a gran escala obtienen importantes mejoras en la eficiencia a consecuencia de la implantación de MLIs. Esto es debido a que, en general, son las grandes empresas las que tienen mayores necesidades de formación específica y mayores dificultades en el seguimiento y control de los trabajadores, y los MLIs permiten poner en marcha estructuras de gobierno para los empleados que solucionan ambos problemas (Siebert y Addison, 1991; Turner, 1994, y De Jorge, 2000).

La cuestión de porqué surgen los MLIs en un periodo caracterizado por grandes conflictos laborales que, en cierta medida, condicionaron la adopción de modelos organizativos de carácter paternalista y autoritario por parte de estas compañías (Comín et al., 1998), se justifica por los beneficios que reporta este sistema tanto a los trabajadores como a los propietarios. Dichos beneficios pueden ser analizados desde el punto de vista del modelo de aversión al riesgo, del modelo de costes de transacción y de la teoría del capital humano específico. Por un lado, los MLIs incrementan la estabilidad laboral de los trabajadores, generalmente considerados adversos al riesgo, reduciendo las incertidumbres derivadas del ciclo económico, de posibles situaciones de incapacidad laboral, etc. (Bailey, 1974). Por otro lado, el interés económico de los MLIs para las grandes compañías se concreta en la disposición de una amplia fuerza de trabajo permanente que permite reducir los costes de transacción y de búsqueda asociados a la continua provisión de

${ }^{3}$ Ya en 1865, los trabajadores de los talleres de MZA habían creado la primera sociedad española de ferroviarios, la denominada Caja de Socorros, que ese mismo año recibió la protección y ayuda económica de la companía, y que en 1871 dio paso a la Sociedad Cooperativa de Consumos y Socorros Mutuos, más extensiva que la anterior, tanto en sus fines como en su proyección (Juez, 1992, p. 327 y pp. 358-359). 
mano de obra con el perfil adecuado a las necesidades de la empresa y, asimismo, posibilita la inversión en capital humano, principalmente mediante formación específica en el puesto de trabajo, con las suficientes garantías de que en el largo plazo dicha inversión revertirá en beneficio de la empresa (Becker, 1962; Siebert y Addison, 1991, y Mackinnon, 2004).

\section{DATOS}

En este artículo se aplican los métodos no lineales de EO con el fin de analizar la información contenida en una amplia base de datos sobre los trabajadores de la compañía ferroviaria MZA, una de las principales concesionarias de este sector en España durante la segunda mitad del siglo XIX y primera del siglo XX. La compañía MZA se estableció en Madrid, en 1856, tras la aprobación de la Ley General de Ferrocarriles, y continuó desarrollando sus actividades hasta 1941, año en el que fue nacionalizada y pasó a formar parte de RENFE. A comienzos del siglo Xx, MZA controlaba más de un tercio del tendido ferroviario nacional y, junto con la "Compañía de los Caminos de Hierro del Norte de España" (Norte), ambas representaban aproximadamente el 75 por cien del empleo total del sector (Anes, 1978, pp. 485-486, y Juez, 1992, p. 109).

Los datos empleados en este estudio proceden del Archivo de la Fundación de los Ferrocarriles Españoles ${ }^{4}$ que, a su vez, los recoge de los registros de las trayectorias laborales correspondientes a los trabajadores de los Talleres Generales de MZA ${ }^{5}$. Esta base de datos contiene información sobre los expedientes de personal conservados de 940 trabajadores que fue-

${ }^{4}$ Estos datos nos han sido generosamente suministrados por Tomás Martínez Vara, profesor de la Universidad Complutense de Madrid.

${ }^{5} \mathrm{Si}$ bien las companías ferroviarias españolas se organizaron en torno a una serie de grandes servicios comunes a todas ellas - administración central, vía y obras, movimiento, tráfico y tracción, servicio este último en el que se integran los talleres generales o principales-, a medida que Norte y MZA se fueron expandiendo, sus organigramas internos se hicieron mucho más complejos (Juez, 1992, p. 88 y pp. 700-701). Así, cada uno de los grandes servicios, dirigido por el correspondiente jefe de servicio, presentaba en estas companías múltiples escalas o servicios de nivel inferior, dirigidos por personal cualificado que contaba con la colaboracion de subjefes, agregados y supervisores. Entre los restantes recursos humanos comunes a los distintos servicios, Juez (1992, pp. 90-93 y p. 176) menciona a los administrativos que, a su vez, se dividían en numerosas categorías, y a un cuantioso grupo de variadas ocupaciones de menor nivel; por último, como personal específico de los talleres se encuentran los operarios, organizados por especialidades y oficios. Aunque no se ha localizado un estudio específico de los Talleres Generales de MZA, un análisis detallado de la jerarquía de puestos y de la distribución de salarios en una entidad equivalente, como son los Talleres Principales de la companía Norte, puede verse en Pérez Sánchez (1992). Los Talleres Generales de MZA se localizaban en Madrid, junto a la estación de Atocha, y desarrollaron sus actividades de mantenimiento y reparación de los ferrocarriles hasta fechas muy posteriores a la integración de esta compañía en RENFE (ver Centro de Estudios Históricos del Ferrocarril Espanol, http://www.cehfe.es/paginas/actuaciones.htm. página consultada el 24/11/2007). 
ron contratados durante el periodo 1882 a 1889 , en plena expansión de la compañía —en 1879, MZA había adquirido la «Compañía de ferrocarriles de Badajoz a Madrid» y posteriormente, en 1898, adquiriría la "Compañía de ferrocarriles de Tarragona a Barcelona y Francia»- ${ }^{6}$. De esta muestra de trabajadores de la compañía MZA, únicamente 533 han servido de base al estudio por no disponer para el resto de una ficha personal suficientemente completa. Una vez depurados los datos, el número total de trabajadores se redujo a 527 .

El problema de los valores perdidos es muy común cuando se analizan grandes bases de datos, como en este caso, y puede abordarse bien ignorando las observaciones incompletas, o bien imputando valores estimados a dichas observaciones. Ambos enfoques presentan inconvenientes: el primero de ellos supone una pérdida de información, mientras que el segundo puede introducir sesgos e incluso generar patrones de comportamiento que no se dan en los datos originales completos ${ }^{7}$. Como ya se ha señalado, la solución adoptada en este estudio ha sido la estimación de los modelos con las fichas de los empleados que presentaban información completa, puesto que se trata de una muestra suficientemente grande y la imputación de datos para una tercera parte de las observaciones podría introducir importantes sesgos en el análisis (Dempster y Rubin, 1987).

Junto con el nombre y apellidos, la base de datos de MZA contiene información sobre la fecha y lugar de nacimiento, empleo para el que fue contratado el trabajador en el momento de incorporarse a la compañía, salario inicial, estado civil, fechas de comienzo y de extinción del contrato del trabajador con la empresa y sus causas. Así pues, la trayectoria laboral de los trabajadores dentro de la compañía tras su contratación no estaría recogida en dicha base de datos. A este respecto, cabe señalar que su contenido es análogo al que presenta la base de datos de la compañía «Canadian Pacific Railways» (CPR), elaborada con el fin de recabar la información necesaria para la creación y gestión de un plan de pensiones para los trabajadores, establecido en 1903, tal y como puede verse en Mackinnon (1998, p. 82). No obstante, el trabajo desarrollado por Mackinnon (1998) para la compañía CPR se centra, básicamente, en el análisis de la edad de incorporación de los trabajadores, sin tener en cuenta la dimensión multivariante de los

\footnotetext{
${ }^{6}$ Durante este periodo, además de estas compañías, MZA adquirió las líneas de ferrocarril de Mérida a Sevilla y de Aranjuez a Cuenca en 1882 y 1883, respectivamente. Estas líneas no entraron en servicio hasta 1885 (Cambó, 1918, t. II, p. 113).

7 Son numerosos los métodos que se han propuesto para tratar de solucionar este problema. Entre ellos se encuentran: imputación de la media, imputación por vecino mas cercano, e imputación por máxima verosimilitud (Myrveit et al., 2001). El problema se complica cuando se trata de valores perdidos en una base de datos que contiene variables tanto de tipo cualitativo como cuantitativo, como es el caso que nos ocupa; una propuesta para estas situaciones es el uso de la metodología del análisis de clase latente (McCutcheon, 1987). Sin embargo, cuando son muchos los valores perdidos - por encima del 10 por cien-, el uso de un método estadístico de imputación puede hacer más mal que bien, tal como apuntan Dempster y Rubin (1987).
} 
datos. En concreto, en este estudio se analizan las ocho variables siguientes: edad de incorporación del trabajador en la compañía, número de años de permanencia en la misma, salario inicial, lugar de nacimiento, estado civil, primer trabajo desempeñado, sección en la que desarrolla su actividad y motivos para la extinción del contrato con la empresa. Mientras que las tres primeras son variables cuantitativas, las cinco últimas tienen naturaleza cualitativa. En las tablas 1 y 2 se resumen los estadísticos descriptivos y las distribuciones de frecuencias de las variables cuantitativas y cualitativas analizadas, respectivamente.

Como puede observarse en la tabla 1, la mitad de los trabajadores fueron contratados por MZA con edades menores o iguales a veintiséis años, acumularon una antigüedad en la compañía menor o igual a los tres años y se incorporaron a la empresa con sueldos iniciales de hasta 2.500 reales anuales. En términos medios, los nuevos trabajadores de MZA percibían en el momento de ser contratados un salario anual de 2.747 reales que, comparado con los salarios medios que percibían los trabajadores de uno de los principales sectores industriales del periodo, como es el sector textil catalán, se encuentra próximo a los salarios de $2.818,4$ y $3.161,6$ reales anuales de la industria de géneros de punto y de la industria algodonera, respectivamente, ambos correspondientes al año 1891 (Llonch, 2004). Asimismo, y dado que la sede de compañía MZA se encontraba en Madrid, es interesante comparar estos salarios con los que percibían en esta localidad los empleados calificados y sin calificar de la construcción que, en términos medios, se situaban en $4.370,5$ y 2.184 reales anuales, correspondientemente ${ }^{8}$

TABLA 1

ESTADÍSTICOS DESCRIPTIVOS DE LAS VARIABLES CUANTITATIVAS INCLUIDAS EN LA BASE DE DATOS

\begin{tabular}{|c|c|c|c|c|c|c|}
\hline \multirow{2}{*}{ Variables } & \multicolumn{2}{|c|}{ Media } & \multirow{2}{*}{ Mediana } & \multirow{2}{*}{ Moda } & \multirow{2}{*}{ Máximo } & \multirow{2}{*}{ Mínimo } \\
\hline & $\begin{array}{c}\text { Esta- } \\
\text { dístico }\end{array}$ & $\begin{array}{l}\text { Error } \\
\text { típico }\end{array}$ & & & & \\
\hline $\begin{array}{l}\text { Edad de incorporación } \\
\text { (años) } \\
\text { Antiguedad en } \\
\text { la empresa (años) } \\
\text { Sueldo inicial (*) } \\
\text { (reales) }\end{array}$ & $\begin{array}{r}27,91 \\
8,88 \\
2.747,00\end{array}$ & $\begin{array}{r}0,36 \\
0,53 \\
37,65\end{array}$ & 2.500 & $\begin{array}{r}23 \\
0 \\
2.250\end{array}$ & $\begin{array}{r}53 \\
52 \\
10.000\end{array}$ & $\begin{array}{r}13 \\
0 \\
500\end{array}$ \\
\hline
\end{tabular}

Fuente: Elaboración propia.

Notas: $\left(^{\star}\right)$ La variable sueldo inicial está expresada en reales de peseta, moneda de curso legal en España en el periodo analizado de 1882 a 1889.

${ }^{8}$ Con el fin de efectuar estas comparaciones salariales, se han anualizado convenientemente los salarios nominales diarios que proporcionan Reher y Ballesteros (1993). 
(Reher y Ballesteros, 1993). En cuanto a los salarios medios percibidos por otros trabajadores de MZA, Juez (1992, pp. 197-198) muestra que en el año 1881 los maquinistas de la compañía percibían entre 12.000 y 8.400 reales anuales, en función de las distintas categorías, y los fogoneros, entre 7.200 y 5.000 reales anuales.

Dada la procedencia de los trabajadores contratados por MZA durante el periodo, la mayoría de ellos procedentes del artesanado urbano y del campesinado, la variable lugar de nacimiento se ha clasificado en cuatro categorías: Madrid, resto de España, Francia y nacidos en otros países. Principalmente son trabajadores de nacionalidad española y la gran mayoría de ellos procedentes de Madrid, sede central de MZA; concretamente un 36,24 por cien del total, tal y como muestra la tabla 2. Diferenciando Madrid del resto de España es posible contrastar si estos últimos ocupaban diferentes empleos a los del resto de españoles, tal vez como consecuencia de posibles diferencias en la formación de estos trabajadores de origen urbano frente a los campesinos o a los trabajadores de la industria rural. MZA fue financiada en parte con capital francés - se constituyó auspiciada por los Rothschild, la sociedad de crédito francesa "Gran Central» y el Marqués de Salamanca-, y el mayor contingente de empleados de la compañía no españoles tenía esta nacionalidad. Este hecho podría obedecer a la mayor cualificación de estos trabajadores, traídos a España por la empresa con el fin de aplicar sus especiales conocimientos, hipótesis que será posteriormente analizada. Por último, nacidos en otros países agrupa a los trabajadores procedentes de una amplia variedad de nacionalidades, desde Cuba hasta Gran Bretaña.

Los diferentes tipos de trabajos desarrollados por los nuevos empleados durante este amplio periodo fueron cuidadosamente anotados en los registros de la compañía. La extensa lista de ellos resulta poco operativa por su amplitud y, en algunos casos, pintoresca. Por ello, la lista detallada de designaciones se ha resumido en tres categorías: en primer lugar, aprendices, peones y ayudantes; en segundo lugar, obreros con cierta cualificación, como son carpinteros, cerrajeros, pintores, albañiles, etc., y, por último, encargados o supervisores. A este respecto es interesante destacar que, coherentemente con el desarrollo de un sistema de MLIs en MZA, durante todo este periodo las ofertas de empleo no incluyen ocupaciones de alto nivel con competencias en la gestión o dirección de la misma, que lógicamente son las mejor remuneradas por la compañía (Martínez-Vara, 2005). Los "puertos de entrada» en MZA son claramente puestos de menor cualificación profesional, también denominados de "cuello azul»; en concreto, un 37 por cien en los niveles de aprendiz, peón y ayudante, y aproximadamente un 62 por cien en el nivel de obrero con cierta cualificación.

Por otra parte, es claro que no puede considerarse totalmente equivalente el empleo de un carpintero y el de un administrativo. Estas diferencias se han tratado de captar mediante una variable que contiene las diversas sec- 
MERCADOS LABORALES INTERNOS EN LA COMPAÑÍA FERROVIARIA MZA, 1882-1889

TABLA 2

DISTRIBUCIÓN DE FRECUENCIAS DE LAS VARIABLES CUALITATIVAS INCLUIDAS EN LA BASE DE DATOS

\begin{tabular}{|c|c|c|c|}
\hline \multirow{2}{*}{ Variables } & \multirow{2}{*}{ Categorias } & \multicolumn{2}{|c|}{ Distribución } \\
\hline & & Frecuencia & Porcentaje \\
\hline Lugar de nacimiento & $\begin{array}{l}\text { Madrid } \\
\text { Resto de España } \\
\text { Francia } \\
\text { Otros países }\end{array}$ & $\begin{array}{r}191 \\
309 \\
16 \\
11\end{array}$ & $\begin{array}{r}36,24 \\
58,63 \\
3,04 \\
2,09\end{array}$ \\
\hline Estado civil & $\begin{array}{l}\text { Soltero } \\
\text { Casado } \\
\text { Viudo }\end{array}$ & $\begin{array}{r}270 \\
246 \\
11\end{array}$ & $\begin{array}{r}51,23 \\
46,68 \\
2,09\end{array}$ \\
\hline Primer empleo & $\begin{array}{l}\text { Aprendiz, Peón o Ayudante } \\
\text { Obrero cualificado } \\
\text { Encargado }\end{array}$ & $\begin{array}{r}195 \\
329 \\
3\end{array}$ & $\begin{array}{r}37,00 \\
62,43 \\
0,57\end{array}$ \\
\hline Sección & $\begin{array}{l}\text { Ajuste } \\
\text { Calderería } \\
\text { Carruajes } \\
\text { Fraguas } \\
\text { Fundición } \\
\text { Guardería } \\
\text { Guarnecido } \\
\text { Instalaciones } \\
\text { Montaje } \\
\text { Oficinas } \\
\text { Pintura y carpintería } \\
\text { Tornos }\end{array}$ & $\begin{array}{r}36 \\
63 \\
137 \\
31 \\
30 \\
3 \\
21 \\
8 \\
54 \\
3 \\
105 \\
36\end{array}$ & \begin{tabular}{r|}
6,83 \\
11,95 \\
26,00 \\
5,88 \\
5,69 \\
0,57 \\
3,98 \\
1,52 \\
10,25 \\
0,57 \\
19,92 \\
6,83
\end{tabular} \\
\hline Causas de baja & $\begin{array}{l}\text { Dimisión } \\
\text { Despido disciplinario } \\
\text { Despido-reducción plantilla } \\
\text { Traslado } \\
\text { Enfermedad } \\
\text { Fallecimiento } \\
\text { Jubilación }\end{array}$ & $\begin{array}{r}145 \\
174 \\
10 \\
37 \\
39 \\
84 \\
38\end{array}$ & $\begin{array}{r}27,51 \\
33,02 \\
1,90 \\
7,02 \\
7,40 \\
15,94 \\
7,21\end{array}$ \\
\hline
\end{tabular}

Fuente: Elaboración propia.

ciones en las cuales se insertan los trabajadores contratados por MZA, como son: ajuste, calderería, carruajes, fraguas, fundición, guardería, guarnecido, instalaciones, montaje, oficinas, pintura-carpintería, y tornos. Como puede observarse en la tabla 2, los trabajadores de esta compañía fueron contratados, en su mayor parte, para realizar tareas propias de los oficios gremiales. A este respecto, Drummond $(1989$, p. 15) señala que el logro de incrementos significativos en el control de los procesos productivos relacionados con este tipo de oficios constituyó un importante problema al que se enfrentó la 
dirección de los talleres de las compañías ferroviarias durante las últimas décadas del siglo XIX, a diferencia del resto de áreas. Esto era debido a que los gremios tenían establecidos métodos tradicionales de trabajo, comúnmente utilizados, al margen de los sistemas internos de las compañías, mientras que en el resto de áreas, éstas imponían sus propias pautas. Para ello, estas empresas abogaron por una mayor división del trabajo en los procesos productivos de los talleres, generada básicamente a través de la burocratización de la gestión de los recursos humanos, que constituye uno de los rasgos característicos de los MLIs (Mackinnon, 1998, p. 80).

En lo relativo al marco institucional vigente, este periodo se inicia con la creación de la Comisión de Reformas Sociales ${ }^{9}$, uno de los referentes básicos de la reforma social española que, en materia de legislación laboral, fue llevada a cabo con un claro retraso con respecto a la mayoría de países europeos ${ }^{10}$. Este hecho parece haber condicionado, en cierta medida, la prolongación de las prácticas empresariales partenalistas en España (Llonch, 2004, p. 104). Dicha Comisión tenía como objetivo el estudio y la propuesta de soluciones a las cuestiones que afectaban a las relaciones entre el trabajo y el capital (Palacio, 1988). Todo ello, con el fin de afrontar la elevada conflictividad laboral existente, elemento que actuó frecuentemente como revulsivo de las iniciativas oficiales en materia de legislación laboral encaminadas a la mejora de las precarias condiciones de trabajo de los obreros, generadas a raíz del proceso de industrialización ${ }^{11}$.

En este contexto, los motivos por los cuales se declara extinguida la relación de los trabajadores con la compañía MZA constituyen un catálogo de las relaciones sociales resultantes de la organización del trabajo y de los conflictos laborales, e incluyen observaciones tales como "provocar desórdenes» o "declararse enfermo mientras está realizando otros trabajos». Todas ellas han sido clasificadas en las categorías: dimisión, despido disciplinario, despido por reducción de plantilla, traslado, enfermedad, muerte y jubilación. Como puede observarse en la tabla 2 el número de dimisiones y despidos entre los nuevos empleados de MZA es muy elevado, ya que ascienden a más del 62 por cien del total. Ahora bien, con la información disponible no es posible establecer los motivos por los cuales se producen en este periodo unas tasas tan elevadas de abandono entre los nuevos tra-

\footnotetext{
9 RD de 5 de diciembre de 1883.

10 La reforma social continuaría con la creación en 1903 del Instituto de Reformas Sociales (RD de 28 de abril de 1903) y culminaría en 1920 con la creación del Ministerio de Trabajo (RD de 8 mayo de 1920).

11 A este respecto, cabe señalar que entre 1872 y 1873 había tenido lugar una oleada de importantes huelgas convocadas por maquinistas y fogoneros de distintas companías ferroviarias. Los siguientes conflictos resenables en este ámbito no se producen hasta 1892 y 1893, con las huelgas de los obreros de los talleres de la compañía Norte y de la «Compañía de Ferrocarriles Andaluces», respectivamente. No fue hasta 1904 cuando los obreros de los Talleres Generales de la compañía MZA llevaron a cabo una huelga, convocada, no obstante, por solidaridad con los mineros de esta misma companía (Juez, 1992, p. 645).
} 
bajadores de MZA - bien sea porque consideraron poco probable llegar a promocionarse dentro de la compañía o bien porque juzgaron que sus oportunidades fuera de la misma serían mejores-, cuestión fundamental que, junto con la alta proporción de despidos, dificultó el establecimiento de un mayor número de relaciones laborales a largo plazo durante este periodo.

En un análisis de la compañía CPR, la principal compañía ferroviaria canadiense, Mackinnon (1998, pp. 80-86) describe una situación similar y nota los efectos adversos de la elevada rotación de los trabajadores sobre los costes de producción, tal y como pone de relieve Fitzgerald (1988, p. 11). Así pues, su reducción constituyó uno de los principales objetivos de la dirección de las compañías ferroviarias y, con esta finalidad, comenzaron a establecer programas de bienestar industrial, más o menos sistematizados ${ }^{12}$. Este modo de proceder es consistente con los planteamientos teóricos de los MLIs (Fitzgerald, 1988, pp. 30-33). A este respecto, Fitzgerald (1988, p. 19; 1999 , p. 173) puntualiza que la progresiva sistematización de los programas de bienestar conduce a mayores reducciones en la rotación de los trabajadores y, consecuentemente, a menores gastos de formación del personal, aunque, no obstante, dicha sistematización estaría condicionada en gran medida por la capacidad de las empresas para garantizar en cualquier circunstancia los beneficios derivados de estos programas.

\section{RESULTADOS}

\subsection{Parámetros de escalado óptimo y componentes principales categóricos}

En la tabla 3 se resumen los parámetros de EO para las distintas categorías de las variables cualitativas contenidas en la base de datos de MZA. Teniendo en cuenta que no se han introducido restricciones de nivel en el algoritmo, es interesante observar la ordenación que, de manera natural, han recibido las distintas categorías mediante la aplicación de este método. Por ejemplo, los parámetros de la variable lugar de nacimiento toman valores especialmente altos para los nacidos en Francia, lo cual es coherente

\footnotetext{
12 Básicamente, en la compañía MZA, estos programas se concretan en la concesión de anticipos sin interés a los trabajadores fijos de la compañía, incluyendo lógicamente al personal de talleres, desde sus primeros tiempos (Ballesteros, 1999, p. 1038) y, asimismo, el servicio médico, el suministro gratuito de medicinas, etc., y los pagos por enfermedad para empleados y obreros con sueldo anual menores de cierto nivel (Juez, 1992, p. 306); la concesion de socorros con motivo de muerte o enfermedad desde la década de 1860 y la caja de previsión, desde 1874 (Juez, 1992, pp. 317-320); la creación de escuelas para los hijos de los empleados, desde 1873 (Juez, 1992, p. 292) y la escuela de formación para futuros empleados (telegrafistas), que se creó en 1883 (Juez, 1992, p. 294); la creación de la caja de pensiones, desde 1900 (Juez, 1992, pp. 317 333 y p. 340); la puesta en marcha de un economato, desde 1902; además de otras ventajas o remuneraciones no salariales que MZA concedía a los trabajadores (Juez, 1992, pp. 217-274).
} 
con la importancia de la presencia francesa en la compañía, según lo apuntado anteriormente.

En la última columna de la tabla 3 puede observarse la alta significación estadística de los parámetros de escalado óptimo, globalmente considerados, para las distintas variables cualitativas. Estos resultados permiten inferir que existen diferencias entre los trabajadores contratados por MZA, diferen-

TABLA 3

PARÁMETROS DE ESCALADO ÓPTIMO QUE CUANTIFICAN LAS VARIABLES CUALITATIVAS Y SIGNIFICACIÓN ESTADÍSTICA

\begin{tabular}{|c|c|c|c|}
\hline \multirow{2}{*}{ Variables } & \multirow{2}{*}{ Categorías } & \multicolumn{2}{|c|}{ Escalado Óptimo } \\
\hline & & Parámetros & $\begin{array}{c}\left.\text { F-estadístico ( }{ }^{(*)}\right) \\
\text { (p-valor) }\end{array}$ \\
\hline Lugar de nacimiento & $\begin{array}{l}\text { Madrid } \\
\text { Resto de España } \\
\text { Francia } \\
\text { Otros países }\end{array}$ & $\begin{array}{r}-0,38 \\
-0,06 \\
5,56 \\
0,57\end{array}$ & $\begin{array}{l}12,708 \\
(<0,01)\end{array}$ \\
\hline Estado civil & $\begin{array}{l}\text { Soltero } \\
\text { Casado } \\
\text { Viudo }\end{array}$ & $\begin{array}{r}-0,97 \\
0,98 \\
1,74 \\
\end{array}$ & $\begin{array}{r}3,716 \\
(<0,01)\end{array}$ \\
\hline Primer empleo & $\begin{array}{l}\text { Aprendiz, Peón o Ayudante } \\
\text { Obrero cualificado } \\
\text { Encargado }\end{array}$ & $\begin{array}{r}-1,30 \\
0,76 \\
1,49\end{array}$ & $\begin{array}{r}26,471 \\
(<0,01)\end{array}$ \\
\hline Sección & $\begin{array}{l}\text { Ajuste } \\
\text { Calderería } \\
\text { Carruajes } \\
\text { Fraguas } \\
\text { Fundición } \\
\text { Guardería } \\
\text { Guarnecido } \\
\text { Instalaciones } \\
\text { Oficinas } \\
\text { Montaje } \\
\text { Pintura y carpintería } \\
\text { Tornos }\end{array}$ & $\begin{array}{r}-0,12 \\
-0,65 \\
0,06 \\
-1,28 \\
-0,86 \\
1,85 \\
0,84 \\
-0,96 \\
1,05 \\
-1,36 \\
1,65 \\
-0,33\end{array}$ & $\begin{array}{l}15,266 \\
(<0,01)\end{array}$ \\
\hline Causas de baja & $\begin{array}{l}\text { Dimisión } \\
\text { Despido disciplinario } \\
\text { Despido-reducción plantilla } \\
\text { Traslado } \\
\text { Enfermedad } \\
\text { Fallecimiento } \\
\text { Jubilación }\end{array}$ & $\begin{array}{r}-0,57 \\
-0,61 \\
-0,83 \\
-0,72 \\
0,94 \\
0,91 \\
2,74\end{array}$ & $\begin{array}{r}20,189 \\
(<0,01)\end{array}$ \\
\hline
\end{tabular}

Fuente: Elaboración propia.

Notas: $\left.{ }^{\star}\right)$ Contraste de la significación estadística global de los parámetros de escalado óptimo, basado en la prueba desarrollada por Fisher (1938). 
cias altamente significativas, que se reflejan en su lugar de nacimiento, el estado civil, el primer empleo, la sección y las distintas causas de baja en la empresa. Como advierte Fisher $(1938,1941)$, este resultado es importante ya que es sólo bajo esta condición cuando los parámetros de EO tienen valor.

Una vez cuantificadas las variables cualitativas, el análisis de CPC pudo realizarse contemplando simultáneamente las ocho dimensiones asociadas a las ocho variables - cinco cualitativas y tres cuantitativas- que incluye esta base de datos. La tabla 4 presenta los valores propios de las dimensiones relevantes obtenidos en la aplicación de dicho análisis, siguiendo el criterio de Jolliffe $(1972,1986)$. Este criterio establece en 0,7 el límite del valor propio, por encima del cual puede considerarse relevante una dimensión - frente al criterio de Kaiser que considera únicamente las dimensiones con valores propios mayores que 1 -, debido a que los errores de muestreo tienden a reducir la magnitud de los valores propios, tal y como subraya Dunteman (1989, pp. 22-23).

TABLA 4

VALORES PROPIOS Y VARIANZA TOTAL EXPLICADA MEDIANTE COMPONENTES PRINCIPALES CATEGÓRICOS

\begin{tabular}{|c|c|c|}
\hline \multirow{2}{*}{ Dimensiones } & \multicolumn{2}{|c|}{ Varianza explicada } \\
\cline { 2 - 3 } & Valores propios & $\%$ Varianza total \\
\hline 1 & 2,178 & 26,29 \\
2 & 1,868 & 49,82 \\
3 & 1,480 & 67,25 \\
4 & 1,037 & 80,28 \\
5 & 0,880 & 90,43 \\
\hline
\end{tabular}

Fuente: Elaboración propia.

Como puede observarse en la tabla 4, cinco valores propios satisfacen la condición de Jolliffe (1972) y explican más del 90 por cien de la variación en los datos. De estos resultados cabe deducir que un empleado de la compañía MZA podría ser descrito adecuadamente a partir de estas cinco características o dimensiones, que se analizan a continuación. Dado que las dos primeras características explican aproximadamente el 50 por cien de la variabilidad en los datos, dichas características constituyen los dos componentes principales más relevantes.

La interpretación de las diferentes dimensiones se basa en el análisis de las saturaciones de las variables, que se resumen en la tabla 5. Como ya es conocido, las variables con mayor ponderación en los componentes son las que los explican en mayor medida. En el primer componente categórico las variables con mayores ponderaciones son el primer empleo, el salario inicial, la edad de incorporación del trabajador en la empresa y el estado civil. 
TABLA 5

TABLA DE SATURACIONES DE LOS COMPONENTES PRINCIPALES CATEGÓRICOS

\begin{tabular}{|l|c|r|r|r|r|}
\hline \multirow{2}{*}{ Variables } & \multicolumn{5}{c|}{ Dimensiones } \\
\cline { 2 - 6 } & $\mathbf{1}$ & $\mathbf{2}$ & $\mathbf{3}$ & \multicolumn{1}{c|}{$\mathbf{4}$} & $\mathbf{5}$ \\
\hline Estado civil & 0,647 & 0,010 & 0,603 & $-0,097$ & $-0,213$ \\
Lugar de nacimiento & 0,227 & $-0,235$ & $-0,166$ & 0,921 & $-0,306$ \\
Edad de incorporación & 0,698 & 0,285 & 0,501 & $-0,017$ & $-0,195$ \\
Primer empleo & 0,715 & $-0,357$ & $-0,461$ & $-0,161$ & 0,145 \\
Sección & 0,407 & $-0,371$ & $-0,538$ & $-0,353$ & $-0,516$ \\
Salario inicial & 0,713 & $-0,082$ & $-0,098$ & 0,159 & 0,643 \\
Causa de baja & 0,182 & 0,863 & $-0,351$ & 0,054 & $-0,045$ \\
Antiguedad & 0,043 & 0,822 & $-0,450$ & 0,023 & $-0,027$ \\
\hline
\end{tabular}

Fuente: Elaboración propia.

Por consiguiente, tendrían valores elevados en este componente los trabajadores con mayor experiencia previa a su incorporación a la empresa, con mayores salarios y para empleos de mayor cualificación profesional, por lo que podría interpretarse como "experiencia y cualificación previas» a la incorporación de los trabajadores en la compañía.

En cuanto al segundo componente categórico, la causa de baja y la experiencia en la empresa son las variables con mayores ponderaciones, por lo que representan la "antigüedad del trabajador en la empresa». Además del estado civil, el tercer componente está asociado con la edad de incorporación y la sección de trabajo, de modo que los que se incorporaron a la empresa a una edad avanzada y trabajaron en las secciones de pintura, tapicería o guardería, tendrían valores elevados en este componente. El cuarto componente está claramente relacionado con el lugar de nacimiento, y el hecho de que esta variable sea un componente en sí mismo indica, además, que no está correlacionada con las restantes variables ni con los otros componentes. Por ello, puede concluirse que el lugar de nacimiento constituye una variable singular que permite diferenciar distintos grupos de trabajadores en la compañía MZA, cuestión que se analiza en el siguiente epígrafe. Por último, en el quinto componente son las variables salario inicial y sección de trabajo las que presentan saturaciones más significativas.

\subsection{Interpretación gráfica de los resultados: análisis Pro-Fit}

El gráfico 1 representa gráficamente a los trabajadores de MZA en las dimensiones que determinan los dos primeros componentes principales categóricos, anteriormente analizados. En dicho gráfico cada punto representa a cada uno de los trabajadores de la compañía y, en la misma, se especifican sus salarios iniciales junto con los correspondientes lugares de nacimiento. 


\section{GRÁFICO 1 \\ REPRESENTACIÓN GRÁFICA DE LOS DOS PRIMEROS COMPONENTES \\ PRINCIPALES CATEGÓRICOS, CON INDICACIÓN DEL SALARIO INICIAL \\ Y EL LUGAR DE NACIMIENTO DE LOS TRABAJADORES DE MZA}

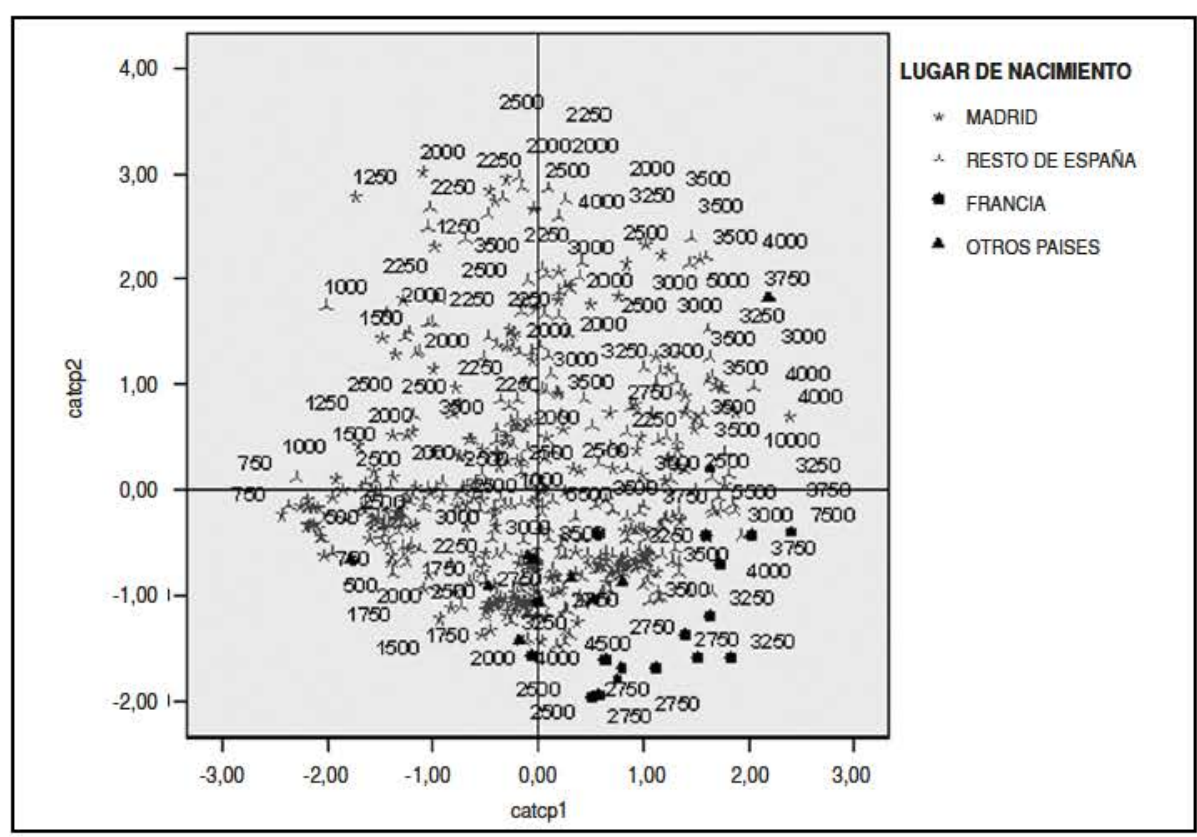

Fuente: Elaboración propia.

Como puede observarse, el salario aumenta de izquierda a derecha, indicando que la edad y la categoría profesional al incorporarse a la compañía - primera dimensión- están claramente relacionadas con el salario inicial percibido por los trabajadores, algo que era de esperar. Esto es precisamente lo que se deriva del sistema de MLIs, ya que los mercados externos influyen en los MLIs a través de los "puertos de entrada» de los trabajadores en la empresa (Wachter y Wright, 1990). También es notorio en el gráfico que los trabajadores nacidos fuera de España se encuentran muy próximos entre sí, lo que indica que comparten características comunes. De su posición en el gráfico 1 se deduce que, con una excepción, los trabajadores no españoles recibieron salarios superiores a la media. Este resultado es coherente con la situación descrita por Juez (1992, p. 76).

Por otro lado, el gráfico 2 muestra nuevamente los datos de MZA en la dimensión de los dos primeros componentes principales, pero en este caso en relación con la causa de cese de los trabajadores en la compañía.

Al analizar las causas del cese de los trabajadores se observa que en la parte superior del gráfico se sitúan aquellos que dejaron la compañía por 
GRÁFICO 2

REPRESENTACIÓN GRÁFICA DE LOS DOS PRIMEROS COMPONENTES

PRINCIPALES CATEGÓRICOS, CON INDICACIÓN DE LA CAUSA DE BAJA Y LA ANTIGUEEDAD DE LOS TRABAJADORES DE MZA

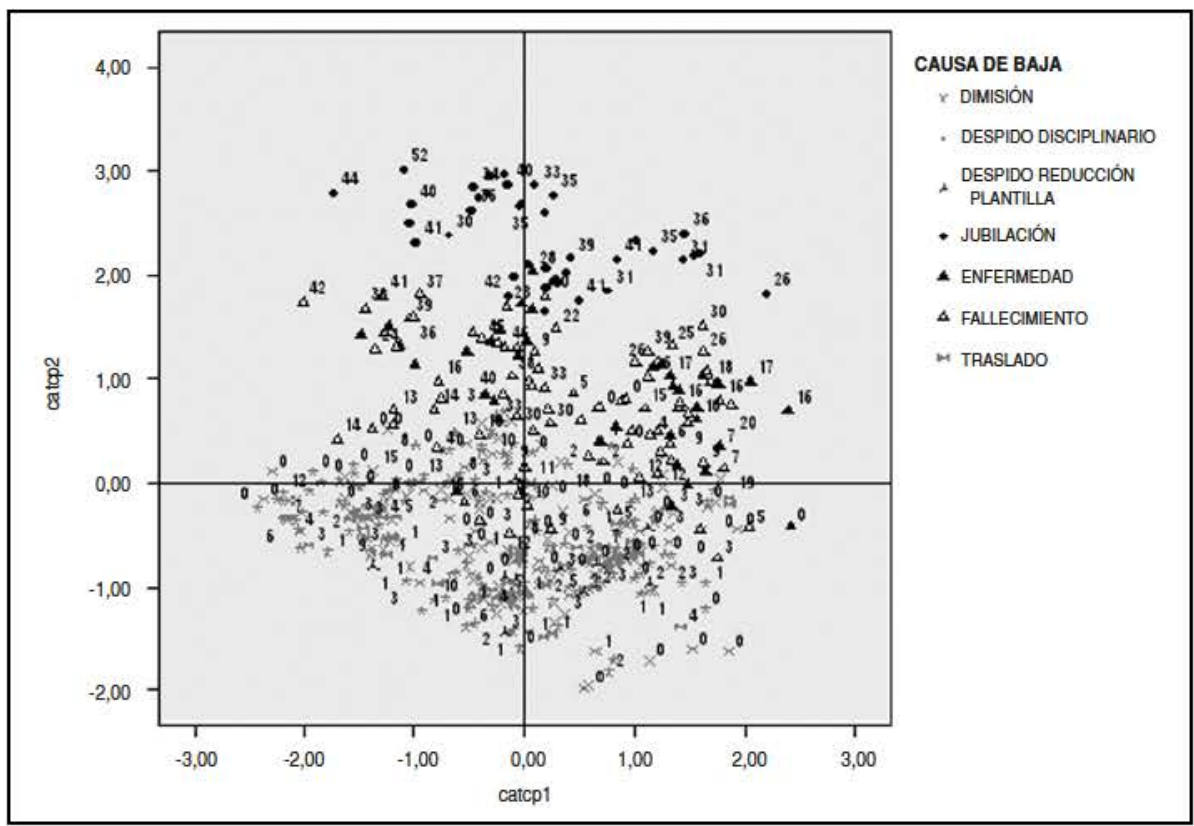

Fuente: Elaboración propia.

causas ordinarias — jubilación, enfermedad, y muerte-, mientras que en la parte inferior se sitúan los que cesaron por razones extraordinarias - reducción de plantilla, despido disciplinario, dimisión y traslado-. Esto sugiere que la conflictividad laboral se concentra en la parte inferior del gráfico y, en este sentido, cabe cuestionarse con qué variables estaría asociada tal conflictividad laboral.

Para explorar estas cuestiones, se ha aplicado el análisis de Ajuste de Propiedades —o Property Fitting (Pro-Fit)—. Inicialmente, Kruskal y Wish (1978) desarrollan está herramienta para variables cuantitativas y, más recientemente, Mar Molinero y Mingers (2007) amplían su ámbito de aplicación, demostrando sus buenas propiedades también para el caso de dicotomías.

El análisis Pro-Fit constituye un método estadístico que se enmarca dentro de las técnicas denominadas Biplots (Gower y Hand, 1996), y permite representar los resultados de un modo gráfico por medio de líneas orientadas, o vectores normalizados (Schiffman et al., 1981). La aplicación del Pro-Fit requiere estimar regresiones auxiliares, donde son variables dependientes las características de los datos que van a relacionarse a partir de la orientación y 
magnitud de los vectores que las representan - como por ejemplo, el salario inicial de los trabajadores, su lugar de nacimiento o la causa de baja-y constituyen los regresores del modelo los valores de los componentes principales categóricos para cada uno de los trabajadores u observaciones. En el caso de variables dependientes cualitativas se ha aplicado la regresión logística.

Los principales resultados obtenidos en el análisis Pro-Fit se resumen en la tabla 6, y en el gráfico 3 se muestran los vectores asociados a las distintas variables analizadas. Al estar basado en la regresión, este método proporciona medidas de la bondad del ajuste y, como puede observarse en dicha tabla, los coeficientes de determinación obtenidos son en todos los casos elevados, de modo que la interpretación de los datos mediante el análisis Pro-Fit resulta adecuada.

TABLA 6

RESULTADOS DEL ANÁLISIS PRO-FIT: COSENOS DIRECCIONALES Y COEFICIENTES DE AJUSTE

\begin{tabular}{|l|r|r|r|r|r|c|}
\hline \multicolumn{1}{|c|}{ Variables } & \multicolumn{1}{c|}{$\boldsymbol{\beta}_{\mathbf{1}}$} & \multicolumn{1}{c|}{$\boldsymbol{\beta}_{\mathbf{2}}$} & \multicolumn{1}{c|}{$\boldsymbol{\beta}_{\mathbf{3}}$} & \multicolumn{1}{c|}{$\boldsymbol{\beta}_{\mathbf{4}}$} & \multicolumn{1}{c|}{$\boldsymbol{\beta}_{\mathbf{5}}$} & $\mathbf{R}^{\mathbf{2}}$ \\
\hline Edad de incorporación & 0.74 & 0.10 & 0.53 & -0.01 & -0.17 & 0.93 \\
Salario inicial & 0.68 & -0.26 & -0.11 & 0.40 & 0.55 & 0.82 \\
Antiguedad $_{\text {Causa de baja }}^{(*)}$ & 0.05 & 0.84 & -0.41 & 0.01 & 0.00 & 0.93 \\
Esto civil $^{(*)}$ & 0.42 & 0.84 & -0.32 & 0.02 & -0.09 & 0.96 \\
Lugar de nacimiento $^{(*)}$ & 0.50 & 0.00 & 0.77 & -0.12 & -0.15 & 0.99 \\
Primer empleo $^{(*)}$ & 0.07 & 0.19 & 0.11 & -0.85 & 0.47 & 0.99 \\
\hline
\end{tabular}

Fuente: Elaboración propia.

Notas: ( $\left.{ }^{\star}\right)$ En los modelos logit se ha incluido el $\mathrm{R}^{2}$ de Nagelkerke.

En el gráfico 3, los vectores que son ortogonales entre sí indican ausencia de correlación entre las variables que representan, mientras que los vectores que forman un ángulo agudo (obtuso) corresponden a variables que están positivamente (negativamente) correlacionadas. En dicho gráfico puede observarse que las variables antigüedad en la empresa y causa de baja están altamente correlacionadas, de modo que quienes pasan muchos años en la empresa la dejan por las causas normales: enfermedad, muerte y jubilación. Por el contrario, los trabajadores que dejan la empresa por causas extraordinarias, esto es, dimisión y despido, suelen ser aquellos que han permanecido en ella poco tiempo.

Este resultado constituye un rasgo característico de la relaciones a largo plazo que se establecen en los MLIs, y es asimismo coherente con las predicciones de la teoría del capital humano específico. Según predice esta teoría, las tasas de dimisión y despido disminuyen a medida que aumenta la antigüedad de los trabajadores en la empresa (Parsons, 1986). Esto no ocurre, sin embargo, en el modelo de compensación diferida, según el cual los 


\section{GRÁFICO 3 \\ ANÁLISIS PRO-FIT: REPRESENTACIÓN GRÁFICA DEL LUGAR \\ DE NACIMIENTO (LN), ANTIGUEDAD EN LA EMPRESA (AE), \\ CAUSA DE BAJA (CB), EDAD DE INCORPORACIÓN (EI), ESTADO CIVIL (EC), SALARIO INICIAL (SI) Y PRIMER EMPLEO (PE)}

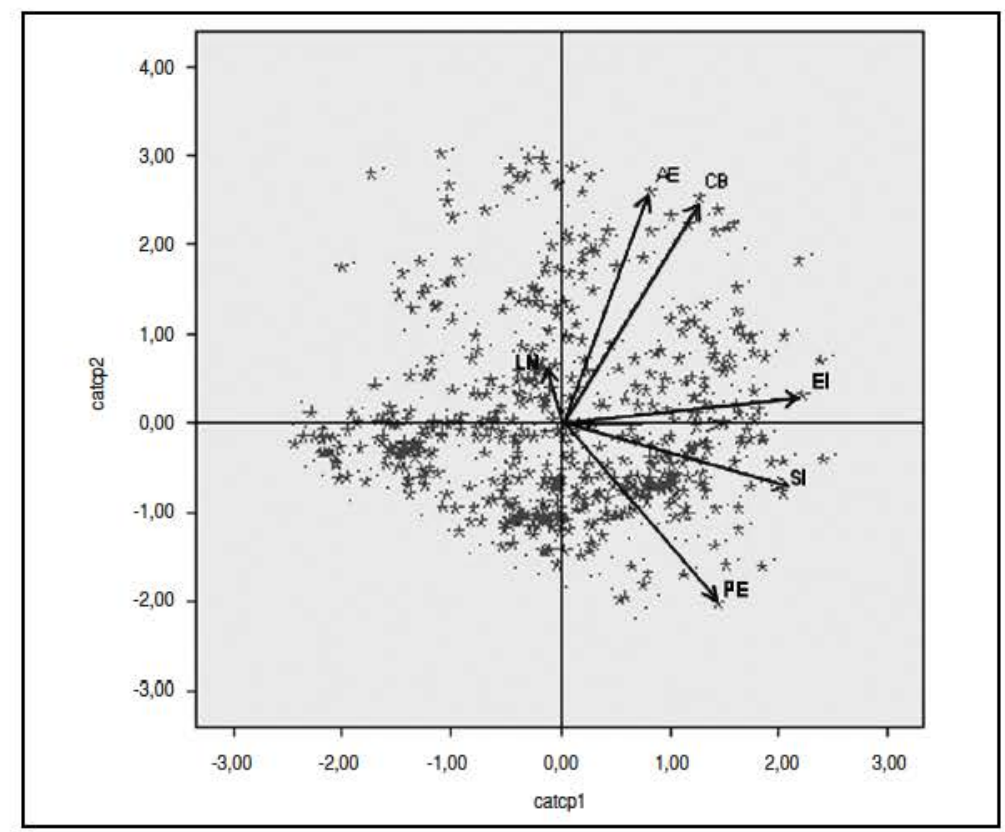

Fuente: Elaboración propia.

salarios superan la productividad marginal de los trabajadores una vez superada la fase de formación específica, y ello desincentiva las dimisiones de los empleados pero incentiva los despidos (Medoff y Abraham, 1981, y Siebert y Addison, 1991).

Por último, el gráfico 3 muestra una correlación positiva entre la edad de incorporación en la empresa y el salario inicial de los trabajadores, coherentemente con lo señalado sobre la influencia de los mercados externos en los internos a través de los "puertos de entrada». Asimismo, es interesante observar que el salario inicial y la antigüedad en la empresa no están asociados - los dos vectores que los representan son prácticamente ortogonales-, como tampoco lo están el salario inicial con la causa de baja. Este resultado podría interpretarse en el sentido de que no fueron las condiciones de entrada de los trabajadores en MZA las que determinaron su permanencia en la misma. Más bien, el elemento clave que explica la permanencia de los nuevos contratados en MZA parecen haber sido las relaciones a largo plazo que se requieren en los MLIs para convertir en beneficios los costes en 
que se incurren en la etapa inicial de incorporación de los trabajadores. Tal y como sugieren la teoría del capital humano, así como el modelo de compensación diferida, las relaciones a largo plazo que se establecen en los MLIs actúan como elemento de auto-selección de los trabajadores más estables (Becker y Stigler, 1974, y Becker, 1975). Esto es debido a que, al compartir trabajadores y empresarios los costes y beneficios de la formación específica, se generan los incentivos adecuados para no interrumpir la relación laboral, una vez establecida, con prematuras dimisiones o despidos. Por otro lado, las estructuras de gobierno de los trabajadores que se desarrollan en los MLIs disuadirían tanto a los trabajadores como a los empresarios de la adopción de comportamientos oportunistas (Wachter y Wright, 1990).

\section{CONCLUSIONES}

En este artículo se han planteado cuestiones de dos tipos: metodológicas y económicas. Empezando por las metodológicas, en el curso de una investigación es habitual disponer de una mezcla de datos cuantitativos y cualitativos. Los métodos no lineales de EO son idóneos en mezclas de este tipo, aunque su aplicación a la economía ha sido poco frecuente. En este estudio se han utilizado los métodos no lineales de EO de Fisher para convertir las variables cualitativas en cuantitativas y se han aplicado contrastes de hipótesis apropiados. La cuantificación de las variables cualitativas ha permitido aplicar métodos estadísticos estándar a una base de datos de empleados de la compañía MZA.

El segundo tipo de cuestiones se relaciona con la presencia de MLIs a finales del siglo XIX en las compañías ferroviarias españolas. Los enfoques teóricos que analizan el sistema de MLIs sugieren que la compañía se relaciona con el mercado laboral exterior a través de unos "puertos de entrada» por los que se contratan a nuevos trabajadores sin formación específica, a los que se les exige un sacrificio inicial que será más tarde recompensado con beneficios tanto salariales como sociales. Estas teorías predicen que la mayor parte de las salidas irregulares de los trabajadores - despidos y dimisiones- se producen tras una corta estancia en la compañía ya que el coste de oportunidad de quienes acumulan cierta antigüedad en la empresa puede ser elevado.

Tras realizar un análisis descriptivo de los datos se observa que gran parte de los contratos se formalizaron en puestos de trabajo de bajos niveles y, asimismo, que no se contrataron especialistas en profesiones específicas de los ferrocarriles. En cuanto a las salidas de la empresa, los resultados muestran que numerosos empleados permanecieron poco tiempo en la compañía. Se observa también la presencia de un contingente importante de empleados de origen francés, lo cual es coherente con la importancia de la presencia francesa en el capital de la compañía y sugiere su posible contratación como especialistas, consultores o asesores. 
La aplicación de las técnicas de EO ha dado como resultado la estimación de unos parámetros altamente significativos en el modelo, cuyos valores se ajustan a las expectativas formuladas a priori. Gracias a estos resultados del EO, pudo realizarse el análisis de CPC contemplando simultáneamente las dimensiones asociadas a todas las variables - cinco cualitativas y tres cuantitativas-que incluye esta base de datos. A partir de este análisis, se han identificado cinco dimensiones significativas en los datos y se ha observado que, para el caso que nos ocupa, basta con examinar las dos primeras, que recogen aproximadamente el 50 por cien de la variabilidad en los datos. Estas dimensiones se han interpretado como experiencia previa y antigüedad en la empresa.

La representación de los datos en el espacio de los dos primeros componentes principales, ampliada mediante el análisis Pro-Fit, permite observar que, en el momento de la contratación inicial, las distintas experiencias se recompensaron con salarios iniciales distintos, tal como sugiere el modelo de costes de transacción aplicado a los "puertos de entrada» en los MLIs. Se ha detectado asimismo una relación directa entre la experiencia y la causa de baja, que confirma que quienes lograron superar el periodo inicial de formación en la compañía tendían a abandonarla por causas normales, como también sugieren otras teorías aplicables a los MLIs. Por otra parte, no se ha observado relación alguna entre el salario inicial y la antigüedad en la empresa, ni tampoco entre el salario inicial y la causa de baja, lo que sugiere que la permanencia en la empresa no fue determinada por las características del contrato inicial sino por el funcionamiento de los MLIs.

En síntesis, tras analizar la posible existencia de alguna forma incipiente de MLI en la compañía ferroviaria MZA en los comienzos de la segunda revolución industrial mediante la aplicación de los métodos no lineales de EO, los resultados permiten concluir que dos rasgos característicos del funcionamiento de dichos MLIs - como son, la existencia de "puertos de entrada» de los trabajadores en la compañía para niveles bajos de cualificación profesional, así como de relaciones laborales que se establecen para el largo plazo - se han detectado en la muestra analizada. Por tanto, análogamente a los resultados que presentan Drummond (1989, 1995); Mackinnon (1998); Savage (1998), y Howlett (2000, 2001, 2004) para el Reino Unido y Australia, concluimos que en España la compañía MZA ya operaba con cierta estructura de MLIs antes del siglo xx.

\section{BIBLIOGRAFÍA}

ANES, R. (1978): «Relaciones entre el ferrocarril y la economía española (1865-1935)», en M. ARTOLa (ed.), Los ferrocarriles en España, 1844-194. Madrid: Servicio de Estudios del Banco de España, pp. 355-512.

Artola, M. (1978): «La acción del Estado», en M. ARTola (ed.), Los ferrocarriles en España, 1844-1943. Madrid: Banco de España, vol. 1, pp. 339-453. 
BAILEY, M. N. (1974): "Wages and unemployment under uncertain demand». Review of Economic Studies, 41 (1), pp. 37-50.

Ballesteros, E. (1999): «La concesión de anticipos sin interés a los empleados de MZA (1857-1875». Anuario Jurídico y Económico Escurialense, 32, pp. 10291046.

Ballesteros, E., y T. Martínez Vara (2001): «La evolución del empleo en el sector ferroviario español, 1893-1935». Revista de Historia Económica, 3, pp. 637-677.

BARLETT, M. S. (1951): "The goodness of fit of a single hypothetical discriminant function in the case of several groups». Annals of Eugenics, 16, pp. 199-214.

Batista-Foguet, J. M.; Fortiana, J.; Currie, C., y Villalbí, J. R. (2004): «Socio-economic Indexes in Surveys for Comparisons between Countries». Social Indicators Research, 67, pp. 315-332.

BECKER, G. S. (1962): «Investment in human capital: A theoretical analysis». The Journal of Political Economy, 70 (5), pp. 9-49.

- (1975): Human capital: A theoretical and empirical analysis. New York: Columbia University Press.

BECKER, G. S., y STIGLER, G. J. (1974): «Law enforcement, malfeasance, and compensation of enforces». Journal of Legal Studies, 3, pp. 1-18.

Самво́, F. (1918): Elementos para el estudio del problema ferroviario en España. Madrid: Ministerio de Fomento.

CARON, F. (2003): "À propos de la rationalisation du travail dans les ateliers des compagnies de chemins de fer en France, 1880-1936». Revue d'Histoire des Chemins de Fer, 28-29, pp. 190-206.

CARRERAS, A., y TAFUNELL, X. (1996): «La gran empresa en la España contemporánea: entre el Mercado y el Estado", en F. COMín y P. MARTín-ACENA (eds.), La empresa en la historia de España. Madrid: Civitas, pp. 73-90.

CASARES, A. (1973): Estudio histórico-económico de las construcciones ferroviarias españolas en el siglo XX. Madrid: Instituto de Desarrollo Económico.

ChandLeR, A. D. (1965): The railroads. The nation's first big business. New York: Harcourt, Brace \& World.

- (1977): The visible hand. The managerial revolution in American business. Cambridge, Mass.: Belknap-Harvard. Hay traducción española de 1988 con el título La mano visible: la revolución en la dirección de la empresa norteamericana. Madrid: Ministerio de Trabajo y Seguridad Social.

Comín, F. (1983): "Comentarios en torno al ferrocarril y el crecimiento económico español entre 1855 y $1931 »$. Revista de Historia Económica, 1, pp. 181-195.

Comín, F.; Martín-Aceñ, P.; Munoz, M., y Vidal, J. (1998): 150 años de historia de los ferrocarriles en España. Madrid: Anaya.

DE JORGE, J. (2000): "Análisis empírico de los mercados internos de trabajo: distribución de salarios y estructura jerárquica de puestos». Cuadernos de Economía y Dirección de la Empresa, 6, pp. 269-284.

De LeEuw, J. (2003): «Least Squares Optimal Scaling of Partially Observed Linear Systems». Department of Statistics, UCLA. Department of Statistics Papers, núm. 2003072701, pp. 1-22.

DE LEeUW, J.; YounG, F. W., y TAKANE, Y. (1976): «Additive structure in qualitative data: an alternating least squares method with optimal scaling features». Psychometrika, 41 (4), pp. 471-503.

Dempster, A. P., y Rubin, D. B. (1983): «Introduction», en A. P. Dempster, I. Olkin y D. B. RuBIN (eds.), Incomplete data sets in sample surveys. New York: Academic Press, vol. 2., pp. 3-10. 
Didow, N. M.; Keller, K. L.; Barksdale, H. C., y FranKe, G. R. (1985): «Improving measure quality by Alternating Least Squares Optimal Scaling». Journal of Marketing Research, vol. XXII, pp. 30-40.

DOERINGER, P. B., y PIORE, M. J. (1971): Internal labour markets and manpower analysis. Lexington, Mass.: Lexington Books, Heath. Hay traducción española de 1985 con el título Mercados internos de trabajo y análisis laboral. Madrid: Ministerio de Trabajo y Seguridad Social.

DRUMmOND, D. (1989): «Specifically designed? Employers' labour strategies and worker responses in British railway workshops, 1838-1914». Business History, 31 (2), pp. 8-31.

- (1995): Crewe: Railway town, company and people, 1840-1914. Aldershot: Scholar Press.

Dunteman, G. H. (1989): Principal Components Analysis. Newbury Park, CA: Sage Publications.

FISHER, R. A $(1938,1941)$ : Statistical methods for research workers (ediciones 7. ${ }^{\mathrm{a}}$ y 8. ${ }^{\mathrm{a}}$ ). Edinburgh: Oliver and Boyd.

FITZgerald, R. (1988): British Labour Management and Industrial Welfare, 1846-1939. London: Croom Helm.

- (1999): «Employment relations and industrial welfare in Britain: Business ethics versus labor markets». Business and Economic History, 28, pp. 167-179.

Gómez MendozA, A. (1982): Ferrocarriles y cambio económico en España entre 1855 y 1913. Madrid: Alianza.

Gower, J. C., y HAND, D. J. (1996): Biplots. London: Chapman \& Hall.

HAYASH, C. (1952): «On the predictions of the phenomena from qualitative data and quantifications of qualitative data from the mathematico-statistical point of viewn. Annals of the Institute of Statistical Mathematics, 3, pp. 69-92.

HERRANZ, A. (2003): «¿Fracasó el sistema ferroviario en España? Reflexiones en torno a la "paradoja del ferrocarril español" ». Revista de Historia Industrial, 23, pp. 39-64.

Howlett, P. (2000): «Evidence of the existence of an internal labour market in the Great Eastern Railway Company, 1875-1905». Business History, 42 (1), pp. 21-40.

- (2001): "Careers for the unskilled in the Great Eastern Railway Company, 18701913». London School of Economics Working Paper Series, 63/01, pp. 1-35.

- (2004): "The internal labour dynamics of the Great Eastern Railway Company, 1870-1913». Economic History Review, LVII (2), pp. 396-422.

IDSON, T., y FEASTER, D. (1990): «A selectivity model of employer-size wage differentials». Journal of Labor Economics, 8 (1), pp. 99-122.

JollifFe, I. T. (1972): «Discarding variables in Principal Components Analysis». Applied Statistics, 21, pp. 160-173.

- (1986): Principal Components Analysis. New York: Springer-Verlag.

JuEZ, E. P. (1992): El mundo social de los ferrocarriles españoles de 1857 a 1917. Tesis Doctoral. Universidad Complutense de Madrid.

KRUSKAL, J. B. (1964): «Multidimensional Scaling by optimizing goodness of fit to a non-metric hypothesis». Psychometrika, 29 (1), pp. 1-27.

KRUSKAL, J. B., y WiSh, M. (1978): Multidimensional Scaling. London: Sage.

LAZEAR, E. P., y OYER, P. (2003): "Internal and external labor markets: a personnel economics approach». NBER Working Paper Series, núm. 10192, pp. 1-50.

LLONCH, M. (2004): "Jornada, salarios y costes labores en el sector textil catalán (18911936)». Revista de Historia Industrial, 26, pp. 101-140.

MACKINNON, M. (1994): «The Great War and the Canadian labour market: railway workers 1903-39", en G. Grantham y M. MacKinNon (eds.), Labour Market Evolution: 
Essays in the Economic History of Market Integration, Wage Flexibility and the Employment Relation. London: Routledge, pp. 205-224.

- (1998): "Careers North and South: Railway Workers in Australia and Canada, 18851940 ", en C. E. NúNEZ (ed.), Railway Management and its Organizational Structure: its Impact on and Diffusion into the General Economy (Proceedings of the Twelfth International Economic History Congress). Sevilla: Universidad de Sevilla, pp. 79-89.

- (2004): «Trade Unions and Employment Stability at the Canadian Pacific Railway, 1903-1929", en D. Mrtch, J. BRown y M. H. D. van LeEuwen (eds.), Origins of the Modern Careers. Aldershot: Ashgate, pp. 126-144.

MAR Molinero, C., y Mingers, J. (2007): «Mapping MBA Programmes: an alternative analysis». Journal of the Operational Research Society, 58, 874-886.

Mar Molinero, C.; Portillo, F., y Hayes, R. (2007): «Analysing the success of a MBA Programme». Kent Business School Working Paper Series, 137, pp. 1-17.

MARTÍNEZ VARA, T. (2005): "Labour cost in a leading Spanish company: MZA Railway Company». Journal of Transport History, 26 (2), pp. 60-78.

- (2006): «Salarios y programas de bienestar industrial en la empresa ferroviaria MZA (1915-1935)». Investigaciones de Historia Económica, 4, pp. 101-138.

McCutcheon, A. L. (1987): Latent class analysis. Newbury Park, California: Sage Publications, Quantitative Applications in the Social Sciences, 64.

MEDOFF, J. L., y ABRAHAM, K. G. (1981): «Are those paid more really more productive? The case of experience». Journal of Human Resources, 16 (2), pp. 186-216.

Meulman, J. J.; VAN DER KoOU, A. J., y HeISER, W. J. (2004): «Principal components analysis with nonlinear optimal scaling transformations for ordinal and nominal datan, en D. KAPLAN (ed.), Handbook of quantitative methodology for the social sciences. Thousand Oaks, CA: Sage Publications, pp. 49-70.

Muñz, M., y VIDAL, J. (2001): "Los ferrocarriles en la historiografía española». Transportes, Servicios y Telecomunicaciones, 1, pp. 81-111.

MyrTVEYT, I.; STENSRUd, E., y OlsSON, U. H. (2001): «Analyzing data sets with missing data: an empirical evaluation of inputation methods and likelihood-based methods». IEEE Transactions in Software Engineering, 27 (11), pp. 999-1013.

NADAL, J. (1975): «El fracaso de la Revolución Industrial en España, 1814-1913». Barcelona: Ariel.

NEAL, P. J., y RoBerTs, G. O. (2006): «Optimal Scaling for partially updating MCMC algorithms». The Annals of Applied Probability, 16(2), pp. 475-515.

ORTÚNEZ, P. P. (1999): «La configuración de la red nacional y las grandes compañías ferroviarias: Norte y MZA, 1913-1936», en M. MunOZ, J. SANZ y J. VidAL (eds.), Siglo y medio del ferrocarril en España 1848-1998. Madrid: Fundación de los Ferrocarriles Españoles, pp. 273-298.

Palacio, J. I. (1988): La institucionalización de la Reforma de España (1883-1924). La Comisión y el Instituto de Reformas Sociales. Madrid: Ministerio de Trabajo y Seguridad Social.

PARSONS, D. O. (1986): «The Employment relationship: Job attachment, work effort and the nature of contracts», en R. LAYARD y O. ASHENFELTER (eds.), Handbook of Labor Economics. Amsterdam: North-Holland, pp. 789-848.

Pérez SÁNCHEZ (1992): "Los Talleres Principales de Reparación de la Compañía del Norte en Valladolid: un estudio de la historia social (1861-1931)». Investigaciones Históricas, 12, pp. 255-283.

Portillo, F.; Mar Molinero, C., y Martínez Vara, T. (2006): «Interpreting a Data Base of Railway Workers using Optimal Scaling Techniques». Kent Business School Working Paper Series, 127, pp. 1-23. 
ReHER, D., y BALlesteros, E. (1993): «Precios y salarios en Castilla la Nueva. La construcción de un índice de salarios reales, 1501-1991 ». Revista de Historia Económica, 1, pp. 101-151.

Sото, А. (1989): El trabajo industrial en la España contemporánea (1874-1936). Barcelona: Anthropos.

SAVAGE, M. (1998): «Discipline, surveillance and the career: Employment on the Great Western Railway 1833-1914», en A. McKINLAY y K. STARKEY (eds.), Foucault, management and organisation theory: Form panopticon to technologies of self. London: Sage, pp. 65-92.

SchiffMAN, S. S.; ReYNolds, M. L., y Young, F. W. (1981): Introduction to Multidimensional Scaling: Theory, Methods and Applications. London: Academic Press.

Serrano, C.; Mar Molinero, C., y Bossi QueIroz, A. (2003): «An approach to the measurement of intangible assets in public sector using scaling techniques». Journal of Intellectual Capital, 4, pp. 249-275.

Serrano, C.; Mar Molinero, C., y Chaparro, F. (2004): «Spanish savings banks: a view on intangibles». Knowledge Management Research \& Practice, 2, pp. 103-117.

SHEN, S. M., y Сноу, S. T. (2005): «The pre- and post- well-being of Hong Kong residents». Social Indicators Research, 71, pp. 231-258.

SIEBERT, W. S., y ADDISON, J. T. (1991): «Internal labour markets: causes and consequences». Oxford Review of Economic Policy, 7 (1), pp. 76-92.

STEVENS, S. S. (1951): "Mathematics, measurement, and psychophysics», en S. S. SteVens (ed.), Handbook of Experimental Psychology. New York: Wiley.

TaKane, Y.; Young, F. W., y De LeEuw, J. (1980): "An individual differences additive model: An Alternating Least Squares method with Optimal Scaling features». Psychometrika, 42, pp. 7-67.

TEDDE, P. (1978): «Las compañías ferroviarias en España (1855-1935)», en M. ARTola (ed.), Los ferrocarriles en España, 1844-1943. Madrid: Banco de España, vol. 2, pp. 9-354.

- (1996): «La expansión de las grandes compañías ferroviarias españolas: Norte, MZA y Andaluces», en F. COMín y P. MARTín-AcENa (eds.), La empresa en la historia de España. Madrid: Civitas, pp. 265-284.

ToRTELLA, G. (1973): «Los orígenes del capitalismo en España. Banca, industria y ferrocarriles en el siglo XIX". Madrid: Tecnos.

- (1999): «La paradoja del ferrocarril español», en M. Muñz, J. SANZ y J. Vidal (eds.), Siglo y medio del ferrocarril en España 1848-1998. Madrid: Fundación de los Ferrocarriles Españoles, pp. 249-253.

TURNER, T. (1994): «Internal labour markets and employment systems». International Journal of Manpower, 15 (1), pp. 15-26.

VIDAL, J. (1996): «La compañías ferroviarias y la difusión de las modernas formas de gestión empresarial en España, 1850-1914», en F. COMín y P. MARTín-ACENA (eds.), La empresa en la historia de España. Madrid: Civitas, pp. 285-301.

- (1997): «La formación de los directivos en la gran empresa: el caso de la Compañía de los Ferrocarriles del Norte de España, 1858-1936». Fundación Empresa Pública, Documentos de Trabajo: Programa de Historia Económica, 9702, pp. 1-119.

- (1999): «La estructura de la propiedad, de la organización y la gestión de una gran empresa ferroviaria: la Compañía de los Caminos de Hierro del Norte de España, 1858-1936». Revista de Historia Económica, 3, pp. 623-662.

WACHTER, M. L., y WRIGHT, R. D. (1990): «The economics of internal labour markets». Industrial Relations, 29 (2), pp. 240-262. 
WeLsh, A. H., y RoBinson, J. (2005): «Fisher and inference for scores». International Statistical Review, 73, pp. 131-150.

YouNG, F. W. (1972): “A model for polynomial conjoint analysis algorithms», en R. N. SHEPARD, A. K. ROMNEY y S. NeRLove (eds.), Multidimensional scaling: Theory and applications in the behavior-sciences. New York: Academic Press, pp. 69-104.

- (1981): "Quantitative analysis of qualitative data». Psychometrika, 46 (4), pp. 357-388.

\section{APÉNDICE}

La idea de asignar valores cuantitativos a conceptos cualitativos mediante los métodos no lineales de EO fue inicialmente planteada por Fisher (1938) -en la séptima edición de Statistical Methods for Research Workers-, con el fin de posibilitar la aplicación de las técnicas estadísticas estándar del análisis multivariante a datos cualitativos. Tras esta aportación inicial, los trabajos de Hayashi (1952), Kruskal (1964), De Leeuw et al. (1976), Takane et al. (1980), De Leeuw (2003), Meulman et al. (2004) y Neal y Roberts (2006), principalmente, han dotado a esta propuesta metodológica de las herramientas necesarias para su aplicación sistemática a numerosos ámbitos de la investigación científica, siendo de especial interés, por su relevancia para el desarrollo de este enfoque, el método de estimación de Mínimos Cuadrados Alternantes (MCA) (Young, 1972).

El principio de los métodos no lineales de EO implica la consideración de las observaciones como categóricas - bien porque éstas posean dicha naturaleza o bien porque sean contempladas como el resultado de un proceso de medida finito-, representando las distintas categorías mediante parámetros cuya optimización conduce a los denominados parámetros de escalado óptimo. Dichos parámetros son el resultado de aplicar una serie de transformaciones a los datos y están sujetos a las restricciones de medida que caracterizan a las variables - por ejemplo, restricciones de orden en variables ordinales - además de las que requiere su normalización. Así pues, desde este punto de vista, los datos consisten en observaciones que se sitúan en una más o menos amplia variedad de categorías, considerándose empíricamente equivalentes las observaciones incluidas en una misma categoría.

El enfoque de Fisher (1938) se enmarca dentro del análisis de la varianza y se basa en el ajuste del modelo aditivo siguiente

$$
Y \beta=X z+\varepsilon,
$$

donde $Y$ representa la $(n \times q)$ matriz indicador normalizada de la variable cualitativa cuyas categorías han de escalarse, $\beta$ el vector de parámetros de EO, $X$ la $(n \times p)$ matriz de diseño normalizada con las restantes variables del modelo, $z$ el habitual vector de parámetros del modelo y $\varepsilon$ el término de error. 
La obtención de los parámetros de $\mathrm{EO}, \beta_{i}$, puede resolverse mediante MCA, que constituye un método de estimación mínimo-cuadrático condicionado. El principio mínimo-cuadrático está condicionado por las estimaciones de los parámetros del modelo y los parámetros de escalado óptimo, que sucesivamente se van alternando como resultado de las iteraciones realizadas hasta alcanzar la transformación óptima de las observaciones. Para ello, es necesario definir una función de pérdida - como pueden ser la suma del cuadrado de los residuos $\mathrm{u}$ otras medidas de disparidad entre las observaciones y las estimaciones, siendo la más habitual el índice de stress de Kruskal (1964) - y optimizar alternativamente con respecto a uno y otro tipo de parámetros, en un proceso iterativo que continua hasta alcanzar el óptimo. Para ello, existen actualmente algoritmos que presentan escasos problemas de mínimos locales (Young, 1981).

Los parámetros de EO pueden obtenerse de forma equivalente a partir de la descomposición del valor singular (DVS) de la matriz de datos

$$
X^{T} Y=\sum_{k=1}^{r} l_{k} a_{k} b_{k}^{T}
$$

donde $r=\min (p, q), a_{k} \in R^{p}$ y $b_{k} \in R^{q}$ y $l_{1} \geq l_{2} \geq \ldots \geq l_{r} \geq 0$. La solución vendría dada por $b_{1}$, el vector singular correspondiente al mayor valor singular de dicha descomposición, $l_{1}$ (ver Welsh y Robinson, 2005). Este enfoque basado en la DVS de los datos facilita además la realización de contrastes de hipótesis sobre los parámetros de EO, cuestión que ha sido abordada de forma relativamente novedosa en este estudio, ya que no se ha identificado ningún otro trabajo en el que se realicen contrastes de hipótesis sobre los parámetros de EO con posterioridad a las aportaciones de Fisher (1938) y Barlett (1951).

Actualmente, esta metodología se ha generalizado de manera que contempla la transformación de cualquier variable cualitativa en variables de naturaleza cuantitativa y, asimismo, su aplicación a una amplia variedad de técnicas de análisis multivariante de datos, como son, la regresión, el análisis de correlación canónica, el análisis discriminante, el análisis de componentes principales y el análisis de correspondencias múltiples. Entre las aplicaciones del escalado óptimo al ámbito de la economía se encuentran Didow et al. (1985), Serrano-Cinca et al. (2003), Batista-Foguet et al. (2004), Serrano-Cinca et al. (2004), Shen y Choy (2005), Portillo et al. (2006) y Mar Molinero et al. (2007). 